\title{
ACCELERATION OF SLOWLY CONVERGENT SE- RIES VIA THE GENERALIZED WEIGHTED-AVERAGES METHOD
}

\author{
A. G. Polimeridis, R. M. Golubović Nićiforović \\ and J. R. Mosig
}

Laboratory of Electromagnetics and Acoustics

Ecole Polytechnique Fédérale de Lausanne

CH-1015 Lausanne, Switzerland

\begin{abstract}
A generalized version of the weighted-averages method is presented for the acceleration of convergence of sequences and series over a wide range of test problems, including linearly and logarithmically convergent series as well as monotone and alternating series. This method was originally developed in a partitionextrapolation procedure for accelerating the convergence of semiinfinite range integrals with Bessel function kernels (Sommerfeld-type integrals), which arise in computational electromagnetics problems involving scattering/radiation in planar stratified media. In this paper, the generalized weighted-averages method is obtained by incorporating the optimal remainder estimates already available in the literature. Numerical results certify its comparable and in many cases superior performance against not only the traditional weighted-averages method but also against the most proven extrapolation methods often used to speed up the computation of slowly convergent series.
\end{abstract}

\section{INTRODUCTION}

Almost every practical numerical method can be viewed as providing an approximation to the limit of an infinite sequence. This sequence is frequently formed by the partial sums of a series, involving a finite number of its elements. Unfortunately, it often happens that the resulting sequence either converges too slowly to be practically useful, or even appears as divergent, hence requesting the use of generalized

Received 7 October 2010, Accepted 28 October 2010, Scheduled 8 November 2010

Corresponding author: Athanasios G. Polimeridis (athanasios.polymeridis@epfl.ch). 
convergence concepts. Indeed, problems with slow convergence or divergence were of course already encountered in the early days of calculus. The idea of applying suitable transformations for the acceleration of the convergence of a series or for the summation of a divergent series is almost as old as calculus itself $[1,2]$. These transformations accomplish this deed by converting a slowly converging or diverging input sequence into another sequence with hopefully better numerical properties.

Sequence transformations can mainly be categorized into linear and nonlinear ones. As typical examples of linear transformations, we mention Euler method [3,4], which is applicable only to alternating series, and the more general-purpose weighted-averages (or generalized Euler) method [5-8]. On the other hand, Shanks transformation [9] and the generalized Levin transformation [10] stand as two of the most representative members of the nonlinear methods. In practical problems, Shanks transformation is efficiently implemented via a recursive scheme, i.e., Wynn's $\epsilon$ algorithm [11], while in Levin transformation, structural information of the sequence is explicitly incorporated in the overall procedure, resulting in some of the most powerful and most versatile sequence acceleration methods that are currently known [12]. Interested readers should consult the excellent monograph [13] for a complete history of previous work relevant to the extrapolation methods.

Although nonlinear transformations stand nowadays as the dominant solution both for mathematical research and practical applications, it is believed that linear transformations could be further improved in order to retain a prominent place in standard mathematical subroutine libraries. Moving towards that direction, a generalized version of the weighted-averages method for the acceleration of slowly convergent series and sequences is presented herein. The basic philosophy of the method was originally introduced in $[14-16]$ for accelerating the converge of Sommerfeld integral tails. This method used the analytic form of the remainder estimates (since the knowledge of the asymptotic behavior of the Sommerfeld-type integrands is usually available), leading to a simple and efficient implementation of the weighted-averages method. According to a recent review paper [17], the resulting algorithm has proven to perform in a comparable or even a better way than the most popular extrapolation methods used for Sommerfeld tails. Often, numerical integration of Sommerfeld integral tails is used as reference for the evaluation of multilayered Green's functions by various other methods $[18,19]$. But the usefulness of this method may go well beyond Sommerfeld integrals as already hinted in the exhaustive monograph 
by Homeier [20].

In this manuscript, focusing on the acceleration of convergence and the summation of series, we present a generalized weighted-averages method together with some simple remainder estimates already available in the literature. The proposed algorithm is of paramount importance for the electromagnetics community, since problems like the efficient computation of $Z$-parameters for the rectangular planar circuit analysis [21] and the MoM solution of the Hallen's equation [22], among many others, call for efficient extrapolation methods without a priori information of the exact remainder estimates. At last, the proposed scheme is compared with some proven extrapolation methods, like $\lambda$-transformation, Levin's transformation with all its variants and $\epsilon$ algorithm of Shanks transformation and the comparison is performed for various series with different types of convergence.

\section{EXTRAPOLATION TECHNIQUES}

The computation of an infinite series

$$
S=\sum_{i=0}^{\infty} u_{i}
$$

with partial sums

$$
S_{n}=\sum_{i=0}^{n} u_{i},
$$

consists in finding the limit of the sequence of those partial sums as $n \rightarrow \infty$, i.e.,

$$
\lim _{n \rightarrow \infty} S_{n}=S
$$

In the case of slowly convergent sequences, though, the remainders

$$
r_{n}=S_{n}-S
$$

do not decay rapidly with increasing $n$, hence, the efficiency of the direct sum is dramatically limited. One way to tackle the aforementioned deficiency and accelerate the convergence of the sequence $\left\{S_{n}\right\}$ is by the means of a transformation (linear or nonlinear) from the original sequence $\left\{S_{n}\right\}$ to a new sequence $\left\{S_{n}^{\prime}\right\}$ with rapidly decaying remainders $r_{n}^{\prime}$.

Numerous series acceleration methods are based on the optimal extraction of the information contained in the sequence of partial sums. The efficiency of the extrapolation method may depend on the type of the convergence of (2) and (4), based on the behavior of the ratio

$$
\lambda_{n}=\frac{S_{n+1}-S_{n}}{S_{n}-S_{n-1}}=\frac{u_{n+1}}{u_{n}} .
$$


More specifically, in the case of convergent series for which the limit of $\lambda_{n}$ exists as $n \rightarrow \infty$,

$$
\lambda=\lim _{n \rightarrow \infty} \lambda_{n},
$$

the convergence is said to be:

- Linear, if $|\lambda| \in(0,1)$

- Sublinear or logarithmic, if $\lambda=1$

- Superlinear or of higher order, if $\lambda=0$

Moreover, the series is asymptotically monotone if $\lambda>0$ and alternating if $\lambda<0$.

Most of the proven extrapolation methods are based on the construction of acceleration algorithms that are exact for the model sequence $[23-25]$

$$
S_{n}=S+\sum_{i=0}^{k-1} a_{i} \psi_{i}(n), \quad n \geq 0, k \geq 1,
$$

where $\psi_{i}(n)$ are known but otherwise arbitrary functions.

For instance, the choice $\psi_{i}(n)=S_{n+i+1}-S_{n+i}$ leads to the (nonlinear) Shanks transformation. The linear system of equations, obtained by writing ( 7$)$ for the indexes $n, \ldots, n+k$ can be solved in terms of determinants using Cramer's rule:

$$
e_{k}\left(S_{n}\right)=\frac{D_{k}\left[S_{n} ; \triangle S_{n+i}\right]}{D_{k}\left[1 ; \triangle S_{n+i}\right]}
$$

where

$$
D_{k}\left[S_{n} ; \Delta S_{n+i}\right]=\left|\begin{array}{ccc}
S_{n} & \ldots & S_{n+k} \\
\Delta S_{n} & \ldots & \Delta S_{n+k} \\
\vdots & \ddots & \vdots \\
\Delta S_{n+k-1} & \ldots & \Delta S_{n+2 k-1}
\end{array}\right| .
$$

Although efficient methods for solving determinants in (8) exist [13], the most convenient way of computing the Shank's transformation is a recursive scheme of $\epsilon$ algorithm given by:

$$
\epsilon_{k+1}^{(n)}=\epsilon_{k-1}^{(n+1)}+\frac{1}{\Delta \epsilon_{k}^{(n)}}, \quad n, k \geq 0
$$

with $\epsilon_{-1}^{(n)}=0, \epsilon_{0}^{(n)}=S_{n}$, and $\Delta$ being the forward difference operator defined by $\Delta \epsilon_{k}^{(n)}=\epsilon_{k+1}^{(n)}-\epsilon_{k}^{(n)}$. If $S_{0}, \ldots S_{2 k}$ are known, $\epsilon_{2 k}^{(0)}$ is the best 
approximation of $S$, while if $S_{0}, \ldots, S_{2 k+1}$ are known $\epsilon_{2 k}^{(1)}$ would be the best approximation of $S$.

On the other hand, by choosing $\psi_{i}(n)=\omega_{n} n^{-i}$ in (7), we get the generalized (nonlinear) Levin transformation. In this case, the remainders for the model sequence are given by

$$
r_{n}=\omega_{n} \sum_{i=0}^{k-1} a_{i} n^{-i}, \quad n \geq 0, k \geq 1,
$$

where $\omega_{n}$ are the associated remainder estimates, which can accommodate explicitly structural information on the $n$-dependence of $r_{n}$, i.e., the behavior of the dominant term of the remainder for large values of $n$. If the remainders of the model sequence (7) are capable of producing sufficiently accurate approximations to the remainders of the sequence $\left\{S_{n}\right\}$, then the application of the sequence transformation to $k+1$ sequence elements should produce a sufficiently accurate approximation to the (generalized) limit $\{S\}$ of the input sequence. Among various options, in cases where the information about the asymptotic behavior of the terms is not available or is difficult to extract, the following four choices of the numerically derived remainder estimates have been suggested as optimal in the literature $[10,26,27]$ :

$$
\begin{aligned}
\omega_{n} & =u_{n+1}, \\
\omega_{n} & =u_{n}, \\
\omega_{n} & =n u_{n}, \\
\omega_{n} & =\frac{u_{n} u_{n+1}}{u_{n+1}-u_{n}} .
\end{aligned}
$$

The above choices yield, respectively, the $t^{\prime}$ transformation of Smith and Ford [7] and the $t, u$ and $v$ transformations of Levin [10]. Although these simple remainder estimates were suggested on the basis of heuristic and asymptotic arguments, they often work remarkably well. More specifically, $t^{\prime}$ and $t$ transformations are capable of accelerating linear convergence and they are particularly efficient in the case of alternating series, but fail to accelerate logarithmic convergence. Levin's $u$ and $v$ transformations, on the other hand, are more versatile since they do not only accelerate linear convergence but also many logarithmically convergent sequences and series [1].

\section{GENERALIZED WEIGHTED-AVERAGES METHOD}

The weighted-averages method [8], or generalized Euler transformation $[6,7]$, or $\lambda$-transformation [5], could be considered as a more so- 
phisticated version of the Euler's transformation, i.e., the simple averaging procedure

$$
S_{n}^{\prime}=\frac{1}{2}\left[S_{n}+S_{n+1}\right]
$$

More specifically, the use of weights for the consecutive partial sums leads to the following formula:

$$
S_{n}^{\prime}=\frac{W_{n} S_{n}+W_{n+1} S_{n+1}}{W_{n}+W_{n+1}} .
$$

Since $S_{n}^{\prime}=S+r_{n}^{\prime}$, the above formula may be written as

$$
S_{n}^{\prime}=S+\underbrace{\frac{W_{n} r_{n}+W_{n+1} r_{n+1}}{W_{n}+W_{n+1}}}_{r_{n}^{\prime}} .
$$

Obviously, the optimal solution would come from the annihilation of the remainders $r_{n}^{\prime}$ of the linearly transformed sequence by imposing an appropriate ratio of the weights,

$$
\eta=\frac{W_{n+1}}{W_{n}}=-\frac{r_{n}}{r_{n+1}} .
$$

Hence, the weighted-averages method could be considered complete if the remainders were explicitly known, which is hardly the case for the sequences of our interest. Next, adopting again the model sequence

$$
S_{n} \sim S+\underbrace{\omega_{n} \sum_{i=0}^{\infty} a_{i} n^{-i}}_{r_{n}}, \quad n \rightarrow \infty,
$$

the ratio of the remainders is given by

$$
\frac{r_{n}}{r_{n+1}}=\frac{\omega_{n}}{\omega_{n+1}}\left[1+O\left(n^{-2}\right)\right], \quad n \rightarrow \infty,
$$

which also suggests that we choose

$$
\eta=-\frac{\omega_{n}}{\omega_{n+1}} .
$$

Then, if

$$
1+\eta=O\left(n^{-\sigma}\right),
$$

the ratio of the remainders for the original and transformed sequence becomes

$$
\frac{\left|r_{n}^{\prime}\right|}{\left|r_{n}\right|} \sim O\left(n^{-p}\right)
$$


where $p=2-\sigma$, manifesting the acceleration property of the aforementioned transformation, when $\sigma<2$. The remainders of the sequence after the transformation have the same form as the original ones together with a scaling factor $n^{-p}$. Finally, the incorporation of the above weights to the weighted-averages transformation (17) leads to the following recursive scheme:

$$
S_{n}^{(k+1)}=\frac{S_{n}^{(k)}+\eta_{n}^{(k)} S_{n+1}^{(k)}}{1+\eta_{n}^{(k)}}, \quad n, k \geq 0,
$$

with

$$
\eta_{n}^{(k)}=-\frac{\omega_{n}}{\omega_{n+1}}\left(\frac{n+1}{n}\right)^{p k},
$$

or the asymptotic form

$$
\eta_{n}^{(k)} \approx-\frac{\omega_{n}}{\omega_{n+1}}\left(1+\frac{p k}{n}\right) .
$$

The recursion (25) represents a triangular scheme,

$$
\begin{array}{ccccc}
S_{0}^{(0)} & S_{0}^{(1)} & \ldots \ldots \ldots \ldots & S_{0}^{(k)} \\
S_{1}^{(0)} & S_{1}^{(1)} & \ldots & S_{1}^{(k-1)} & \\
S_{2}^{(0)} & \ldots & S_{2}^{(k-2)} & & \\
\vdots & . \cdot & & \\
S_{k}^{(0)} & & &
\end{array}
$$

where in the first column $S_{n}^{(0)}=S_{n}$ and $S_{0}^{(k)}$ is the best approximation of $S$, given the partial sums $S_{0}, \cdots, S_{k}$. This generalized version of the weighted-averages method was introduced in [14-16] with a further elaboration in [17], basically for the computation of the Sommerfeld integral tails, as already mentioned in the introduction. It is also referred in the literature as the Mosig-Michalski algorithm, arising as a special case of $J$ transformation [20].

Since the generalized weighted-averages method was first developed for speeding-up the convergence of oscillatory slowly converging integrals, special attention was paid to efficiently use information about the asymptotic behavior of the integrand for that type of problems. In the case where information about the asymptotic behavior is not available or is extremely difficult to extract, simple remainder estimates can be alternatively calculated. In this manuscript, we utilize the remainder estimates (9)-(12) depending on the type of the convergence. The specific choice gives rise to four 
different variants of the generalized weighted-averages method, which will be referred to as $\mathrm{WA}_{\mathrm{I}}, \mathrm{WA}_{\mathrm{II}}$, $\mathrm{WA}_{\mathrm{III}}$ and $\mathrm{WA}_{\mathrm{IV}}$, respectively. Moreover, the two different options for $\eta_{n}^{(k)}$, (26) and (27), lead respectively to the "exact" and "asymptotic" form of the algorithm. It is easy to prove that for series with associated remainders as in (20) and the aforementioned remainder estimates, $\sigma=0 \rightarrow p=2$ for all cases, except for the logarithmically (monotone) converging series, where $\sigma=1 \rightarrow p=1$ only for $\mathrm{WA}_{\mathrm{III}}$ and $\mathrm{WA}_{\mathrm{IV}}$. Finally, note that Euler and $\lambda$-transformation (or simple weighted-averages method) are special cases of (25) when $\eta=1$ and $\eta=-\lambda$, respectively.

\section{NUMERICAL RESULTS}

In this section, a selection of numerical results, including both alternating and monotone infinite series, will be presented for the comparison of the generalized weighted-averages method with some of the most popular and powerful linear and nonlinear transformations.

\subsection{Alternating Series}

More specifically, in the case of the following alternating series:

$$
\sum_{n=0}^{\infty} \frac{(-1)^{n}}{\sqrt{n+1}}=(1-\sqrt{2}) \zeta(0.5)
$$

and

$$
\sum_{n=0}^{\infty} \frac{(-1)^{n}}{2 n+1}=\frac{\pi}{4},
$$

where $\zeta(\cdot)$ is the Riemann zeta function, the proposed algorithm is compared with $\lambda$-transformation, $\epsilon$ algorithm of Shanks transformation and generalized Levin transformation. Note, that for all the numerical results reported herein, we keep the best candidates among all the possible variants of generalized weighted-averages method and generalized Levin transformation. Based on the behavior, i.e., significant digits of the answer versus number of iterations $(k)$, as depicted in both Figs. 1(a) and 1(b), we could say that the method proposed in this manuscript seems to outperform even the $u$ transformation of Levin, which is considered to be among the most versatile and powerful convergence accelerators currently known [7]. It is important to mention also that for reasons unknown, using the asymptotic form of the coefficients $\eta_{n}^{(k)}$ given by (27) results in a faster convergence than using the exact form (26). 


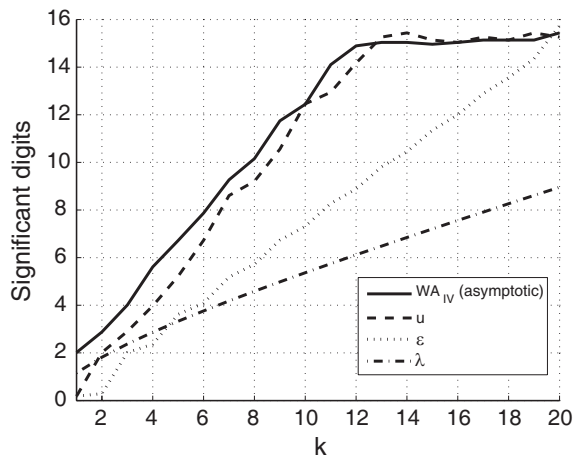

(a) Test example (29)

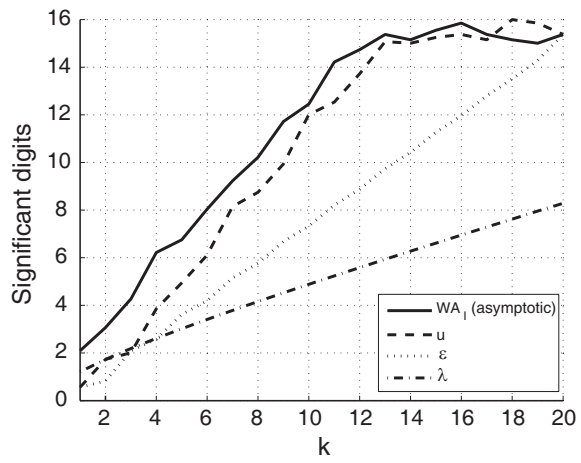

(b) Test example (30)

Figure 1. Performance of various extrapolation methods in computing alternating series.

\subsection{Monotone Series}

Moving to monotone series, we report some representative numerical results, often used for testing the efficiency of extrapolation methods. For the sake of clarity, we choose to split the test problems in two groups according to the type of convergence, i.e., logarithmic and linear.

\subsubsection{Logarithmically Convergent Series}

The first group of test problems consists of the monotone logarithmically convergent series. More specifically, we consider the following two series:

$$
\sum_{n=0}^{\infty} \frac{1}{(n+1)^{2}}=\frac{\pi^{2}}{6}
$$

and

$$
\sum_{n=0}^{\infty} \ln \left(\frac{n+2}{n+1}\right) \cdot \ln \left(\frac{n+3}{n+2}\right)=\ln (2) \ln (K),
$$

where $K$ is the Khinchin's constant. As shown in Fig. 2, where we discarded both $\lambda$-transformation and $\epsilon$ algorithm of Shanks transformation since the fail on accelerating logarithmic monotone series, the behavior of generalized weighted-averages method and generalized Levin transformation is comparable. 


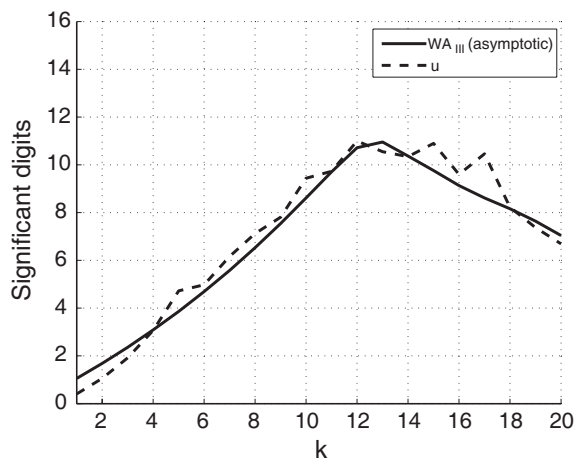

(a) Test example (31)

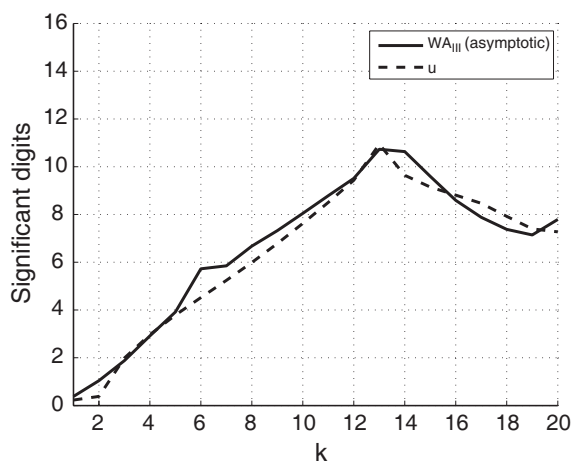

(b) Test example (32)

Figure 2. Performance of extrapolation methods in computing logarithmically convergent (monotone) series.

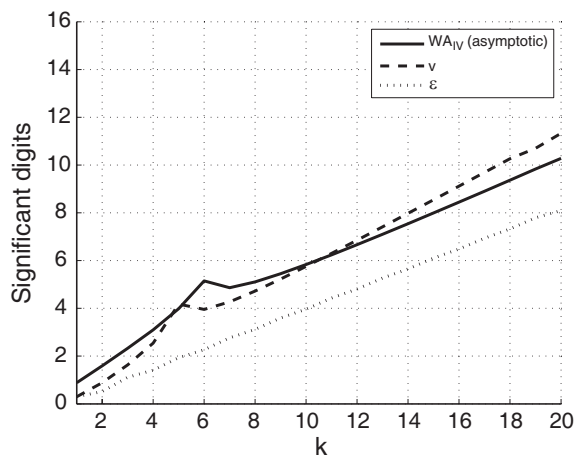

(a) Test example (33)

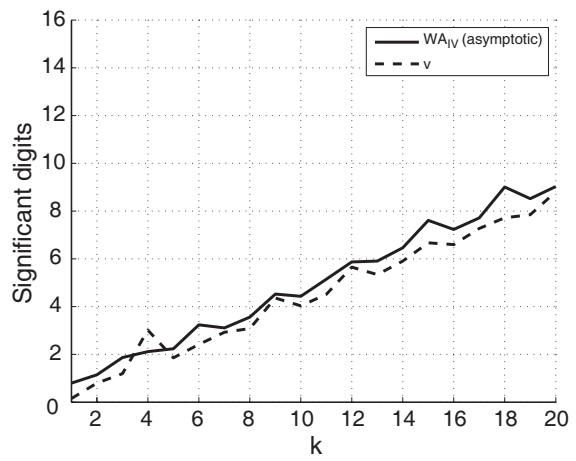

(b) Test example (34)

Figure 3. Performance of various extrapolation methods in computing linearly convergent (monotone) series.

\subsection{Linearly Convergent Series}

The second group of test problems consists of the monotone linearly convergent series,

$$
\sum_{n=0}^{\infty} \frac{(0.8)^{n+1}}{n+1}=\ln (5)
$$

and

$$
\sum_{n=0}^{\infty}\left[(0.4)^{n}+(0.8)^{n}\right]=\frac{20}{3} .
$$


Again, by a simple inspection of Fig. 3, it is easy to come to the conclusion that generalized weighted-averages method and generalized Levin transformation are behaving comparable, while outperforming the $\epsilon$ algorithm of Shanks transformation.

\section{CONCLUSION}

In this paper, we present a generalized version of the weighted-averages method for accelerating slowly convergent (alternating and monotone) sequences and series. The proposed algorithms utilize the remainder estimates that have been already incorporated in the different variants of the generalized Levin transformation. A series of representative numerical results reported in this study comes to verify the noteworthy behavior of the generalized weighted-averages method. In fact, this novel linear transformation is capable of outperforming even the most powerful and versatile nonlinear transformations, that are widely used in various numerical problems.

\section{ACKNOWLEDGMENT}

The authors would like to thank COST Actions IC0803-RFCSET and IC0603-ASSIST for supporting this work.

\section{REFERENCES}

1. Weniger, E. J., "Nonlinear sequence transformations: Computational tools for the acceleration of convergence and the summation of divergent series," preprint arXiv:math/0107080v1, http://arXiv.org.

2. Valagiannopoulos, C. A., "An overview of the Watson transformation presented through a simple example," Progress In Electromagnetics Research, Vol. 75, 137-152, 2007.

3. Longman, I. M., "Note on a method for computing infinite integrals of oscillatory functions," Proc. Cambridge Phil. Soc., Vol. 52, 764-768, 1956.

4. I'A, T. J., An Introduction to the Theory of Infinite Series, Macmillan, New York, 1965.

5. Scraton, R. E., "A note on the summation of divergent power series," Proc. Cambridge Phil. Soc., Vol. 66, 109-114, 1969.

6. Wynn, P., "A note on the generalized Euler transformation," Computer J., Vol. 14, 437-441, 1971. 
7. Smith, D. A. and W. F. Ford, "Acceleration of linear and logarithmic convergence," SIAM J. Num. Anal., Vol. 16, 223-240, 1979.

8. Drummond, J. E., "Convergence speeding, convergence and summability," J. Comput. Appl. Math., Vol. 11, 145-159, 1984.

9. Shanks, D., "Nonlinear transformation of divergent and slowly convergent sequences," J. Math. Phys., Vol. 34, 1-42, 1955.

10. Levin, D., "Development of nonlinear transformations for improving convergence of sequences," Int. J. Comput. Math., Section B, Vol. 3, 371-388, 1973.

11. Wynn, P., "On a device for computing the $e_{m}\left(S_{n}\right)$ transformation," Math. Tables Aids Comput., Vol. 10, 91-96, 1956.

12. Smith, D. A. and W. F. Ford, "Numerical comparisons of nonlinear convergence accelerators," Math. Comput., Vol. 38, 481499, 1982.

13. Brezinski, C. and M. R. Zaglia, Extrapolation Methods, Amsterdam, North-Holland, 1991.

14. Mosig, J. R. and F. E. Gardiol, "A dynamical radiation model for microstrip structures," Adv. Electron. Electron Phys., Academic Eds, New York, Vol. 59, 139-237, 1982.

15. Mosig, J. R. and F. E. Gardiol, "Analytical and numerical techniques in the Green's function treatment of microstrip antennas and scatterers," Proc. Inst. Elect. Eng., Vol. 130, 175$182,1983$.

16. Mosig, J. R., "Integral equation techniques," Numerical Techniques for Microwave and Millimeter-wave Passive Structures, T. Itoh Eds, 133-213, Wiley, New York, 1989.

17. Michalski, K. A., "Extrapolation methods for Sommerfeld integral tails," IEEE Trans. Antennas and Propagat., Vol. 46, 1405-1418, 1998.

18. Li, H., H.-G. Wang, and H. Zhang, "An improvement of the GeEsselle's method for the evaluation of the Green's functions in the shielded multilayered structures," Progress In Electromagnetics Research, Vol. 88, 149-161, 2008.

19. Firuzeh, Z. H., G. A. E. Vandenbosch, R. Moini, S. H. H. Sadeghi, and R. Faraji-Dana, "Efficient evaluation of Green's functions for lossy half-space problems," Progress In Electromagnetics Research, Vol. 109, 139-157, 2010.

20. Homeier, H. H. H., "Scalar Levin-type sequence transformations," J. Comput. Appl. Math., Vol. 122, 81-147, 2000.

21. Liu, P. and Z.-F. Li, "Efficient computation of $Z$-parameter for 
the rectangular planar circuit analysis," PIERS Online, Vol. 1, No. 5, 611-614, 2005.

22. Fikioris, G., "An application of convergence acceleration methods," IEEE Trans. Antennas and Propagat., Vol. 47, 17581418, 1999.

23. Schneider, C., "Vereinfachte rekursionen zur Richardsonextrapolation in spezialfällen," Num. Math., Vol. 24, 177-184, 1975.

24. Håvie, T., "Generalized Neville type extrapolation schemes," BIT, Vol. 19, 204-213, 1979.

25. Brezinski, C., "A general extrapolation algorithm," Num. Math., Vol. 35, 175-180, 1980.

26. Sidi, A., "A user-friendly extrapolation method for oscillatory infinite integrals," Math. Comput., Vol. 51, 249-266, 1988.

27. Fessler, T., W. F. Ford, and D. A. Smith, "HURRY: An acceleration algorithm for scalar sequences and series," $A C M$ Trans. Math. Software, Vol. 9, 346-354, 1983. 